\title{
Sexual Behaviors in Women with Primary and Secondary Provoked Vestibulodynia: A Controlled Study
}

\author{
Bernard Lambert $^{1^{*}}$, Mylène Desrosiers ${ }^{2}$, Miguel Chagnon ${ }^{3}$, Yves Lepage ${ }^{3}$ \\ ${ }^{1}$ Department of Obstetrics-Gynaecology CHUM Montreal, Montreal, Canada \\ ${ }^{2}$ Department of Psychology, University of Montreal, Montreal, Canada \\ ${ }^{3}$ Department of Mathematics and Statistics, University of Montréal, Montreal, Canada \\ Email: *bernard-lambert@videotron.ca; mylene.desrosiers@umontreal.ca; chagnon@dms.umontreal.ca, lepage@dms.umontreal.ca
}

Received May 12, 2013; revised June 12, 2013; accepted June 20, 2013

Copyright @ 2013 Bernard Lambert et al. This is an open access article distributed under the Creative Commons Attribution License, which permits unrestricted use, distribution, and reproduction in any medium, provided the original work is properly cited.

\begin{abstract}
Introduction: Provoked vestibulodynia affects $12 \%$ of the general female population and more specifically, $21 \%$ of women aged less than 30 years. Primary and secondary vestibulodynia are hypothesized to represent the endpoints of different etiologic pathways, although there is still little research addressing potential distinctions between these two groups, particularly with regard to sexuality. Aims: To compare sexual activity and behavior of women with provoked primary vestibulodynia (PVD1) and secondary vestibulodynia (PVD2) against age-matched controls. Methods: Fiftyseven participants $(\mathrm{N}=57)$, mean age $25.72(18$ - 41) recruited from a gynecology clinic underwent a gynaecological examination and completed a self-report questionnaire: $20(\mathrm{~N}=20)$ were diagnosed with primary provoked vestibulodynia (PVD1), 19 ( $\mathrm{N}=19)$ with secondary provoked vestibulodynia (PVD2), and 18 ( $\mathrm{N}=18$ ) were medically confirmed as no-pain controls. Main outcome: To verify any differences in the sexual behavior between primary, secondary vestibulodynias and controls. Results: Mean pain duration differed significantly in participants with PVD1 at 73.8 months against those with PVD2 at 37.4 months $(p=0.003)$. Frequency of sexual activity also differed significantly between the three groups $(\mathrm{p}=0.012)$ : the controls were at $27.8 \%$ against $0 \%$ in primary and secondary vestibulodynias for once or more a day. No significant difference was observed for the sexual arousal time and masturbation frequency. Vaginal penetration was overrepresented in controls $(p<0.001)$ contrary to fellatio frequency $(p=0.016)$. Pain digital test was significantly different between the three groups in one finger (3.85 vs 0.08$)$, two fingers ( 4.39 vs 0.06 ) or three fingers (5.39 vs 0.56) (PVD1 against controls), lubricated inserted fingers for pain verification ( $<<0.001)$. Conclusions: Provoked vestibulodynia generates problems in the sexual response and coital activity, this syndrome reflecting absence of pre-existing sexual problems, notably in the masturbatory activity and oral receptive female sex.
\end{abstract}

Keywords: Primary and Secondary Provoked Vestibulodynia; Sexual Behavior; Vulvar Pain; Sexual Frequency; Vaginal Intromission

\section{Introduction}

Provoked vestibulodynia and pain by vaginal intromission is a frequent problem involving $21 \%$ of the 18 - 29 years females [1] and $12 \%$ of the general female population [2] where vaginal atrophy involving cervicovaginal cul-de-sacs, mucosa thinning and endopelvic fascia shrinking is excluded [3]. Surgical control of provoked vestibulodynia selected cases by vestibulectomy and resection of the posterior vestibular mucosa from 8 to 4 for o'clock has been applied, when the anterior vestibule was not involved in the pain process. However, Bornstein $[4,5]$ observed different datas in primary versus secon-

${ }^{*}$ Corresponding author. dary vestibulodynias, as if it represented two different entities. We previously studied results from the two groups of vestibulodynias, and surprisingly found no significant improvement in surgical treated primaries cases for preoperwith the secondaries controlled at $7.2 \pm 1.3$ preoperative and $2.9 \pm 3.1$ postoperative $(\mathrm{p}<0.001)$ [6], as noticed by Bohm-Starke [7].

It is with this rationale, that we studied sexual habits of women who suffered from primary versus secondary provoked vestibulodynia versus age-matched controls, in order to determine possible differences in the sexual activity and sex comprehension of these women. Secondaries appeared more frequent, two against one, when compared to primaries, and slightly older than primaries. 
Our goal was to evaluate possible differences in regard with the sexual function, attitudes toward sexuality as well as impaired sexual functioning $[8,9]$. The other and main challenge was to verify the sexual behaviors in the two groups, in comparison with a control sample of agematched patients. One important concern, was to evaluate if PVD1 exhibited more conservative sexual behavior and thinking, than PVD2 and controls. The absence of comparison before and since the emergence of provoked vestibulodynia in primaries, who always suffered of painful intromission, and unsatisfactory intercourse, is particularly challenging.

We performed evaluation of the sexual habits in the PVD1 and PVD2 in comparison with controls, especially covering sexual attitudes, in the sexual behavior and practices.

\section{Material and Methods}

Participants were 20 women diagnosed with primary provoked vestibulodynia (PVD1), 19 women with secondary provoked vestibulodynia (PVD2) and 18 controls. This research was approved by the Ethics Committee of the CHUM hospital and was held from January to December 2012. Women who suffered from provoked vestibulodynia (PVD1 and PVD2) were recruited at the vulvar pain clinic and were examined by the same physician (BL). The standard examination conduct by this physician in order to diagnose provoked vestibulodynia, consisted in the 12 hour Q-tip test noted on a scale 0 - 10, and insertion in the lower vagina of one, two, three lubricated fingers with registration of provoked pain test 0 - 10. If an induced pain of 7 - 8 with one or two inserted finger was observed, the examination was immediately stopped. After the examination, women who received a diagnosis of PVD1 or PVD2 were informed by the physician of the existence of the study. Women who were interested to know more about this research were met in private by a research assistant, who explained the process and gave consent form and questionnaires, which have to be filled at home and returned by mail. Women in the control group were recruited via announcements on billboards at the Université de Montréal. Interested women contacted the research coordinator, and an appointment was planned with the physician (BL) for the standard gynaecological examination. Then, the same procedure was applied.

Participants who suffered of provoked vestibulodynia (PVD1 and PVD2) met the following inclusion criteria: 1) aged between 18 and 45 years of age, 2) pain during sexual intercourse from at least six months being a source of subjective distress and occurring in $75 \%$ of penetrative attempts, 3) absence of any spontaneous pain or vulvodynia, and 4) having a diagnosis of provoked insertive or intromission vestibulodynia by the physician
(BL). For the age-matched control group, the inclusion criteria were the following: 1) aged between 18 and 45 years of age, 2) absence of any sexual pain history like vulvar, vaginal or pelvic pain and 3) being sexually active. The exclusion criteria were 1) vulvar pain unrelated to sexual intercourse or vestibular area pressure, 2) pregnancy, 3) severe psychiatric or medical disorder, and 4) active vaginal infection, 5) any vulvar pathology, such as fungus cyclical vaginitis, eczema, lichen sclerosus, planus or simplex, 6) vulvodynia, 7) vaginism or 8) any deep coital or abdominal pain.

Women who were taking part of this study received a questionnaire package, covering sociodemographic information, pain history, pain intensity (0 - 10), sexual history since coitarche, and sexual behaviors. The sexual behaviors questionnaire used in this study include 18 items adapted from the NHSLS Questionnaire used by Laumann and colleagues [1] in their extensive study of the sexual practices of Americans. This brief adaptation of this questionnaire, developed for the purposes of this study, served to measure different aspects of women's sexuality in the past 12 months, such as the number of sexual partners, frequency of sexual activities, type of sexual practice, presence of sexual thoughts, and presence or absence of guilt about some aspects of sexuality.

\section{Statistical Analysis}

A Pearson chi square test was performed to compare the distribution of the discrete variables. For the continuous variables, a one way analysis of variance with a factor at three levels was used to compare the means of the three groups; when significant results were obtained, Tukey's contrasts were used to compare the means of the groups two by two. Finally, for the comparison of the means between the groups and the vestibular sides (anterior and posterior), a two way analysis of variance with repeated measures on one of the factors, a factor group with three level and a repeated factor side with two levels (anterior and posterior) was employed; if necessary, Tukey’s contrasts were used for pairwise comparisons.

\section{Results}

Mean age of the three groups PVD1 was 23.90 years ( $\mathrm{N}$ = 20), PVD2 26.95 years $(\mathrm{N}=19)$ and controls $26.44(\mathrm{~N}$ $=18$ ) years did not differ significatively $(p=0.117$, Table 1), as well as scolarity and age at coitarche. Legal status did not differ significantly between the groups ( $\mathrm{p}=$ 0.104).

However mean pain duration differed significantly (p $=0.003$ ) between primaries and secondaries (73.85 months in primaries versus 37.47 months in secondaries, Table 1). Sexual frequency, did differ significantly between the three groups ( $p=0.012)$; sexual frequency of 
Table 1. Sample characteristics $\mathbf{N}=\mathbf{5 7}$.

\begin{tabular}{cccccc}
\hline Variables & $\begin{array}{c}\text { Primaries } \\
\mathbf{N = \mathbf { 2 0 }}\end{array}$ & $\begin{array}{c}\text { Secondaries } \\
\mathbf{N = 1 9}\end{array}$ & $\begin{array}{c}\text { Controls } \\
\mathbf{N = \mathbf { 1 8 }}\end{array}$ & $\begin{array}{c}\text { Total } \\
\mathbf{N}=\mathbf{5 7}\end{array}$ & $\mathbf{p}$ \\
\hline Age & $23.90(20-31)$ & $26.95(18-41)$ & $26.44(20-38)$ & $25.72(18-41)$ & 0.117 \\
Education (years of scolarity) & $15.25(8-21)$ & $16.89(12-24)$ & $17.17(11-23)$ & $16.40(8-24)$ & 0.104 \\
Age of first intercourse & $17.58(14-21)$ & $16.83(14-20)$ & $17.28(14-25)$ & $17.24(14-25)$ & 0.595 \\
Pain duration months & $73.85(24-180)$ & $37.47(6-96)$ & ---- & $56.13(6-180)$ & 0.003 \\
\hline
\end{tabular}

once or more daily is more frequent in the control group (Table 2). The duration of sexual stimulation $(p=0.101)$ and masturbation frequency ( $\mathrm{p}=0.468$ ) were not significantly different between the three groups (Tables $\mathbf{3}$ and 4). Orgasm during masturbation between the three groups was also not significantly different $(p=0.527)$ [10].

According to the expectations, the frequency of vaginal intromission was highly significant between the groups ( $p<0.001$ ); the frequency of "sometimes" for the primaries was $42.1 \%$ compared to $0 \%$ for the controls while the frequency of "always" in the controls was at $55.6 \%$ against $5.3 \%$ for the primaries (Table 5). For fellatio (Table 6), there is a significant difference between the three groups ( $\mathrm{p}=0.016)$; the added frequency of always and usually for the primaries is $79.0 \%$ compared to $33.3 \%$ for the controls. Anal penetration was significant ( $p=0.025$ ) in the frequency repartition of sometimes, rarely and never for primaries, against secondaries and controls, representing a possible alternative solution, for the primaries vaginal painful intromission (Table 7).

Finally the digital test pain was confirmed as discriminant and significant, between the groups ( $\mathrm{p}<0.001)$ for one, two, and three fingers in pain provocation, the controls tolerating easily with no distress, a three-finger vaginal dilatation (pain for controls at 0.56 vs. 3.11 in PVD2, and 5.39 in OVD1), and also for one and two finger insertion (Table 8).

For vestibular pain mapping, there is no significant mean difference between the sides (anterior and posterior) independently of the groups $(p=0.123)$ but, independently of the side, there is a significant mean difference between the groups ( $p<0.001)$; the mean for the primaries is greater than the mean of the secondaries ( $\mathrm{p}=$ 0.024 ) and the controls ( $p<0.001$ ), and the mean pain for the secondaries is greater than the mean pain for the controls $(\mathrm{p}=0.003)$.

\section{Discussion}

This study gives an interesting lighting on the interference, consequent to the continuous sexual pain, prejudicial to couple interrelations, and absence of enjoyment during vaginal sexual intercourse, this leading to a diminution of the sexual frequency and vaginal intromission.
No primary negative attitudes were observed in the auto-sexual gratification for all groups, underlining the absence of a primary aversion to this type of sexual activity. The consequence of pain hinders the ability to reach gratifying pleasure, essential for obtaining the expected soothing orgasm. Anxiety levels and psychosexual differences in primaries associated with dys-menorrhea and lower systolic blood pressure, in contrast with local vulvar pathology for secondaries has been reported by Granot [11], but more research in this field is needed. On the other side, vulvar tissue status, neural hyperplasia, pain receptors, mast cells have also been reviewed in regard of significant histologic features [12, 13].

The primary provoked vestibulodynia remains a serious challenge and enigma for the practising gynecologist and physician, in regard to the three involved axis: cerebral mapping, vestibule histology and sexual habits, resulting from painful and no gratifying sexual activity, with no whatsoever, anterior comparison, such as in the secondary vestibulodynias. Moreover, the anorgasmic patient is relatively non eligible for anesthetic vestibular desensitization with xylocaine, lidocaine, prilocaine, and ketamine ointments, and sexual therapy appears mandatory in these patients as a first line of treatment. At the other end of the scale, more research is needed for the evaluation of the cerebral mapping, structural brain imaging, and gray matter density in affected young women [14-16].

This small pilot study demonstrates the importance of a parallel sexual evaluation by a sex therapist and treatment in physical therapy, when attempting the temporary (two-three months) limited no vaginal penetration, this being followed by a period of sexual transition and training of the female in a superior sex position, where the penetration is actively controlled by the patient, in order to lower the intromission pain anxiety, and preferably after the female orgasm; this represents an essential condition before attempting normal and various sexual positions. The permission and encouragement for auto-sexuality had been initially granted for personal stimulation (sex for one) followed by a shared non penetrative sexual stimulation, during the two-three month 
Table 2. Sexual activity frequency.

\begin{tabular}{|c|c|c|c|c|}
\hline & Primaries & Secondaries & Controls & Total \\
\hline 1/day or > & $0(0 \%)$ & $0(0 \%)$ & $5(27.8 \%)$ & $5(9.1 \%)$ \\
\hline 3 - 6/weekly & 2 (10.5\%) & 3 (16.7\%) & $4(22.2 \%)$ & 9 (16.4\%) \\
\hline 1 - 2/weekly & $8(42.1 \%)$ & $5(27.8 \%)$ & $6(33.8 \%)$ & 19 (34.5\%) \\
\hline 2 - 3/monthly & 8 (42.1\%) & 5 (27.8\%) & $2(11.1 \%)$ & 15 (27.3\%) \\
\hline 1/monthly or less & $1(5.3 \%)$ & $5(27.8 \%)$ & $1(5.6 \%)$ & $7(12.7 \%)$ \\
\hline
\end{tabular}

$\mathrm{p}=0.012$. Significant $\mathrm{p}$ indicates a difference in sexual activity frequency between groups once a day or more at $0 \%$ in PVD1-2 against $27.8 \%$ in controls.

Table 3. Sexual stimulation duration (in minutes).

\begin{tabular}{rcccc}
\hline & Primaires & Secondaires & Témoins & Total \\
\hline No stimulation & $0(0 \%)$ & $2(11.8 \%)$ & $0(0 \%)$ & $2(3.7 \%)$ \\
$\mathbf{1}-\mathbf{5}$ minutes & $3(15.8 \%)$ & $6(35.3 \%)$ & $4(22.2 \%)$ & $13(24.1 \%)$ \\
$\mathbf{5}-\mathbf{1 0}$ minutes & $5(26.3 \%)$ & $6(35.3 \%)$ & $10(55.6 \%)$ & $21(38.9 \%)$ \\
$\mathbf{1 0}-\mathbf{1 5}$ minutes & $8(42.1 \%)$ & $2(11.8 \%)$ & $3(16.7 \%)$ & $13(24.1 \%)$ \\
$>\mathbf{1 5}$ minutes & $3(15.8 \%)$ & $1(5.9 \%)$ & $1(5.6 \%)$ & $5(9.3 \%)$ \\
\hline
\end{tabular}

$\mathrm{p}=0.101$.

Table 4. Masturbatorysrequency.

\begin{tabular}{ccccc}
\hline & Primaries & Secondaries & Controls & Total \\
\hline A few times weekly & $5(25 \%)$ & $3(16.7 \%)$ & $5(27.8 \%)$ & $13(23.2 \%)$ \\
Once weekly & $3(15.0 \%)$ & $2(11.1 \%)$ & $3(16.7 \%)$ & $8(14.3 \%)$ \\
$\mathbf{2}-\mathbf{3}$ times monthly & $6(30 \%)$ & $4(22.2 \%)$ & $3(16.7 \%)$ & $13(23.2 \%)$ \\
Once a month & $2(10 \%)$ & $0(0.0 \%)$ & $2(11.1 \%)$ & $4(7.1 \%)$ \\
Once/2 months & $0(0.0 \%)$ & $1(5.6 \%)$ & $1(5.6 \%)$ & $2(3.6 \%)$ \\
$\mathbf{3}$ - $\mathbf{\text { times a year }}$ & $0(0.0 \%)$ & $0(0.0 \%)$ & $2(11.1 \%)$ & $2(3.6 \%)$ \\
$\mathbf{1}-\mathbf{2}$ times a year & $1(5 \%)$ & $4(22.2 \%)$ & $1(5.6 \%)$ & $6(10.7 \%)$ \\
Never this year & $3(15 \%)$ & $4(22.2 \%)$ & $1(5.6 \%)$ & $8(14.3 \%)$ \\
Total & $20(100 \%)$ & $18(100.0 \%)$ & $56(100.0 \%)$ & $5 \%)$ \\
\hline
\end{tabular}

$p=0.468$.

Table 5. Vaginal intromission.

\begin{tabular}{ccccc}
\hline & Primaries & Secondaries & Controls & Total \\
\hline Always & $1(5.3 \%)$ & $2(11.8 \%)$ & $10(55.6 \%)$ & $13(24.1 \%)$ \\
Usually & $4(21.1 \%)$ & $8(47.1 \%)$ & $7(38.9 \%)$ & $19(35.2 \%)$ \\
Sometimes & $8(42.1 \%)$ & $3(17.6 \%)$ & $0(0 \%)$ & $11(20.4 \%)$ \\
Rarely & $3(15.8 \%)$ & $4(23.5 \%)$ & $1(5.6 \%)$ & $8(14.8 \%)$ \\
Never & $3(15.8 \%)$ & $0(0.0 \%)$ & $0(0.0 \%)$ & $3(5.6 \%)$ \\
Total & $19(100 \%)$ & $17(100 \%)$ & $18(100 \%)$ & $54(100 \%)$ \\
\hline
\end{tabular}

$\mathrm{p}<0.001$. 
Table 6. Fellatio.

\begin{tabular}{ccccc}
\hline & Primaries & Secondaries & Controls & Total \\
\hline Always & $4(21.1 \%)$ & $1(5.6 \%)$ & $0(0.0 \%)$ & $5(9.1 \%)$ \\
Usually & $11(57.9 \%)$ & $4(22.2 \%)$ & $6(33.3 \%)$ & $21(38.2 \%)$ \\
Sometimes & $4(21.1 \%)$ & $9(50.0 \%)$ & $8(44.4 \%)$ & $21(38.2 \%)$ \\
Rarely & $0(0.0 \%)$ & $4(22.2 \%)$ & $2(11.1 \%)$ & $6(10.9 \%)$ \\
Never & $0(0.0 \%)$ & $0(0.0 \%)$ & $2(11.1 \%)$ & $2(3.6 \%)$ \\
Total & $19(100 \%)$ & $18(100.0 \%)$ & $18(100 \%)$ & $55(100.0 \%)$ \\
\hline
\end{tabular}

$\mathrm{p}=0.016$.

Table 7. Anal penetration.

\begin{tabular}{ccccc}
\hline & Primaries & Secondaries & Controls & Total \\
\hline Sometimes & $2(10.5 \%)$ & $0(0.0 \%)$ & $0(0.0 \%)$ & $2(3.6 \%)$ \\
Rarely & $1(5.3 \%)$ & $1(5.6 \%)$ & $6(33.3 \%)$ & $8(14.5 \%)$ \\
Never & $16(84.2 \%)$ & $17(94.4 \%)$ & $12(66.7 \%)$ & $45(81.8 \%)$ \\
Total & $19(100 \%)$ & $18(100 \%)$ & $18(100 \%)$ & $55(100 \%)$ \\
\hline
\end{tabular}

$p=0.025$.

Table 8. Digital test pain/10.

\begin{tabular}{cccccc}
\hline & Primaries & Secondaries & Controls & Total average & $\mathbf{p}$ \\
\hline $\mathbf{N}$ & 20 & 19 & 18 & 57 & \\
One finger & 3.85 & 1.00 & 0.06 & 1.70 & $<0.001$ \\
Two fingers & 4.39 & 2.17 & 0.06 & 2.03 & $<0.001$ \\
Three fingers & 5.39 & 3.11 & 0.56 & 2.54 & $<0.001$ \\
\hline
\end{tabular}

period of analgesic control of the vestibular and the Hart zone. This can be appraised and comprised by the patients, as a temporary sexual step, and alternative, toward an integral, enriched, and shared sexuality.

\section{Conclusions}

Provoked vestibulodynia primary or secondary is not the result of disturbed sexual attitudes or comprehension, but on the contrary, consequent to defense mechanisms generated by continuous penetrative pain, disturbing the sexual activity frequency, vaginal intromission, and anal penetration practices.

More research is needed in the field of vulvar pain receptors and local inflammatory reactions, as well as the cerebral imaging and the response to pain of provoked vestibulodynias in order to understand the mechanisms of this current condition.

\section{REFERENCES}

[1] E. O. Laumann, A. P aik and R. C. Rosen, "Sexual Dysfunction in the United States: Prevalence and Predictors,"
Journal of the American Medical Association, Vol. 281, No. 6, 1999, pp. 537-544. doi:10.1001/jama.281.6.537

[2] B. L. Harlow, L. A. Wise and E. G. Stewart, "Prevalence and Predictors of Chronic-Lower Genital Tract Discomfort," American Journal of Obstetrics \& Gynecology, Vol. 185, No. 3, 2001, pp. 545-550. doi:10.1067/mob.2001.116748

[3] A. Kao, Y. M. Binik, R. Amsel, D. Funaro, N. Leroux and S. Khalifé, "Challenging Atrophied Perspectives on Postmenopausal Dyspareunia: A Systematic Description and Synthesis of Clinical Pain Characteristics," Journal of Sex \& Marital Therapy, Vol. 38, No. 2, 2012, pp. 128150. doi:10.1080/0092623X.2011.569641

[4] J. Bornstein, Z. Goldzik, Z. Stolar, D. Zarfati and H. Abramovovici, "Predicting the Outcome of Surgical Treatment of Vulvar Vestibulitis," Obstetrics \& Gynecology, Vol. 89, No. 5, 1997, pp. 695-698. doi:10.1016/S0029-7844(97)00102-6

[5] J. Bornstein, M. Maman and H. Abramovici, "Primary vs Secondary Vulvar Vestibulitis. One Disease, Two Variants," American Journal of Obstetrics \& Gynecology, Vol. 184, No. 2, 2001, pp. 28-31. doi:10.1067/mob.2001.108173

[6] B. Lambert, S. Bergeron, M. Desrosiers and Y. Lepage, 
"Introital Primary and Secondary Dyspareunia: Multimodal Clinical and Surgical Control,” Sexologies, Vol. 21, No. 1, 2012, pp. 9-12. doi:10.1016/j.sexol.2011.10.001

[7] N. Bohm-Starke and E. Rylander, "Surgery for Localized, Provoked Vestibulodynia: A Long-Term Follow-Up Study," Journal of Reproductive Medicine, Vol. 53, No. 2, 2008, pp. 83-89.

[8] G. Desrochers, S. Bergeron, T. Landry and M. Jodoin, "Do Psychosexual Factors Play a Role in the Etiology of Provoked Vestibulodynia? A Critical Review,” Journal of Sex \& Marital Therapy, Vol. 34, No. 3, 2008, pp. 198226. doi:10.1080/00926230701866083

[9] K. S. Sutton, C. F. Pukall and S. Chamberlain, "Pain, Sexual and Psychophysical Characteristics of Women with Primary vs Secondary Provoked Vestibulodynia," The Journal of Sexual Medicine, Vol. 6, No. 1, 2009, pp. 205214. doi:10.1111/j.1743-6109.2008.01038.x

[10] “The Hite Report,” MacMillan Publishing Co., New York, 1976.

[11] M. Granot, M. Friedman, D. Yarnitsky, A. Tamir and E. Z. Zimmer, "Primary and Secondary Vulvar Vestibulodynia Syndrome. Systemic Pain Perception and Psychophysical Characteristics," American Journal of Obstetrics
\& Gynecology, Vol. 191, No. 1, 2004, pp. 138-142. doi:10.1016/j.ajog.2003.09.060

[12] C. M. Leclair, M. F. Goetsch, V. B. Korcheva, R. Anderson, D. Peters and T. K. Morgan, "Differences in Primary Compared Secondary Vestibulodynia by Immunochemistry,” Obstetrics \& Gynecology, Vol. 117, No. 6, 2011, pp. 1307-1313. doi:10.1097/AOG.0b013e31821c33dc

[13] M. Goetsch, T. K. Morgan, V. B. Korcheva, H. Li, D. Peters and C. M. Leclair, "Histologic and Receptors Analysis of Primary and Secondary Vestibulodynia and Controls: A Prospective Study,” American Journal of Obstetrics \& Gynecology, Vol. 202, No. 614, 2010, pp. e1-8.

[14] P. Schweinhardt, A. Kuchinad, C. F. Pukall and M. C. Bushnell, "Increased Gray Matter Density in Young Women with Chronic Vulvar Pain,” Pain, Vol. 140, No. 3, 2008, pp. 411-419. doi:10.1016/j.pain.2008.09.014

[15] A. May, "Structural Brain Imaging: A Window into Chronic Pain,” Neuroscientist, Vol. 17, No. 2, 2011, pp. 209220. doi: $10.1177 / 1073858410396220$

[16] P. M. Di Notto, L. Newman, S. Wall and G. Einstein, "The Hermunculus: What Is Known about the Representation of the Female Body in the Brain?” Cerebral Cortex, 2012. 